\title{
Status of the international Muon ionization cooling experiment
}

\section{MAURIZIO BONESINI*广}

Sezione INFN Milano Bicocca

E-mail: maurizio.bonesiniemib.infn.it

The international Muon Ionization Cooling Experiment (MICE) will carry out a systematic investigation of ionization cooling of a muon beam. The demonstration comprises one cell of the neutrino factory cooling channel. As the emittance measurement will be done on a particle-byparticle basis, sophisticated beam instrumentation is needed to measure particle coordinates and timing vs RF. A PID system, in order to keep beam contamination $(e, \pi)$ well below $1 \%$, based on time-of-flight stations, two Aerogel Cerenkov detectors and a KLOE-like calorimeter has been constructed and installed at RAL. It will be supplemented soon by two magnetic spectrometers based on scintillating-fiber trackers and a second downstream calorimeter. The experiment will be performed in steps. In phase I, currently under way, the beamline and the detector diagnostic system will be assembled and the beam characteristics studied. Phase II will add the cooling channel components and allow the quantitative study of ionization cooling. The goal of the MICE Collaboration is to complete the experiment by 2012 .

European Physical Society Europhysics Conference on High Energy Physics

July 16-22, 2009

Krakow, Poland

${ }^{*}$ Speaker.

on behalf of the MICE Collaboration 


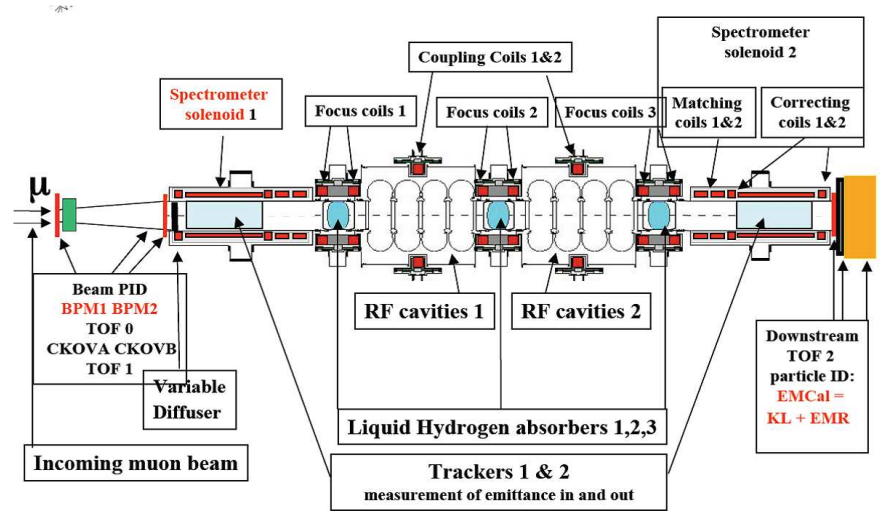

Figure 1: 2-D layout of the MICE experiment at RAL. The beam enters from the left. The cooling section is put between two magnetic spectrometers and two TOF stations (TOF1 and TOF2) to measure particle parameters.

\section{Introduction}

The neutrino factory $(v F)[1]$ is a muon storage ring with long straight sections, where decaying muons produce collimated neutrino beams of well defined composition and high intensity. Several $v \mathrm{~F}$ designs have been proposed, such as the ones of references [2, 3, 4]. The physics performance of a Neutrino Factory depends not only on its clean beam composition $\left(50 \% v_{e}, 50 \% \bar{v}_{\mu}\right.$ for the $\mu^{+} \mapsto \bar{v}_{\mu} v_{e} e^{+}$case), but also on the available beam intensity [5]. The cooling of muons (accounting for $\sim 20 \%$ of the final costs of the factory) is thus needed, increasing the performance up to a factor 10. Different $v \mathrm{~F}$ designs require a muon cooling factor from 2 to 16 , over a distance of $\sim 100 \mathrm{~m}$. Due to the short muon lifetime $(2.2 \mu \mathrm{s})$, novel methods such as the ionization cooling [6] must be used. The cooling of the transverse phase-space coordinates of a muon beam can be accomplished by passing it through a light energy-absorbing material and an accelerating structure, both embedded within a focusing magnetic lattice. Longitudinal and transverse momentum are lost in the absorber while the RF-cavities restore only the longitudinal component. The MICE experiment [7] at RAL (see figure 1 for the layout) aims at a systematic study of a section of the cooling channel of the proposed US Study 2 [4], attaining a $10 \%$ effect for a $6 \pi \cdot \mathrm{mm}$ rad beam.

As conventional emittance measurement techniques reach barely a $\sim 10 \%$ precision, a novel method based on single particle measurements has been envisaged. Particles are measured before and after the cooling section by two magnetic spectrometers complemented by TOF detectors. For each particle $\mathrm{x}, \mathrm{y}, \mathrm{t}, \mathrm{x}^{\prime}=\mathrm{dx} / \mathrm{dz}=p_{x} / p_{z}, \mathrm{y}^{\prime}=\mathrm{dy} / \mathrm{dz}=p_{y} / p_{z}, \mathrm{t}^{\prime}=\mathrm{dt} / \mathrm{dz}=E / p_{z}$ coordinates are measured. In this way, for an ensemble of $\mathrm{N}$ particles, the input and output emittances may be determined with a precision up to $0.1 \%$, that allows the extrapolation of the results to a full cooling channel.

\section{The MICE beamline and cooling channel}

The secondary muon beam from ISIS (140-240 MeV/c central momentum, tunable between 1$10 \pi \cdot \mathrm{mm}$ rad input emittance) enters the cooling section after a $\mathrm{Pb}$ diffuser of adjustable thickness (see figure 2 for details). A Ti target [8] dips into the halo of the primary ISIS proton beam a few milliseconds before its extraction. The produced pions are then captured by a triplet of quadrupoles, followed by a dipole for momentum selection. The $\pi$ 's then decay in a $5 \mathrm{~m}$ long, 12 


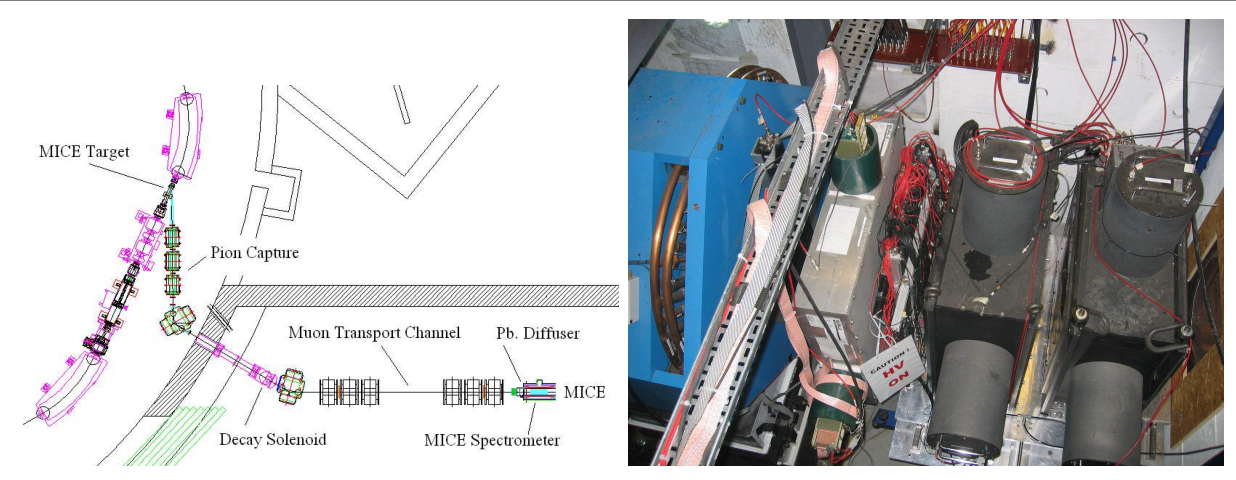

Figure 2: Left: layout of the MICE secondary beamline. $800 \mathrm{MeV} / \mathrm{c}$ protons from ISIS impinge on a Ti target. Produced pions are then guided to a decay solenoid, where muons are then steered to the MICE apparatus. The upstream detectors TOF0 and CKOVa/b are inserted between the two triplets of quads. Right: picture of the installed TOF0,CKOVa,CKOVb upstream detectors after the first quadrupoles triplet.

$\mathrm{cm}$ bore superconducting (SC) decay solenoid, giving muons that enter the cooling section, which is equipped with detectors for emittance measurements on a particle by particle basis. The entire beamline has been designed to give about 600 muons per target shot [9]. All the beamline elements have been installed and commissioned in 2008 , but problems with the target and the cooling decay solenoid have delayed its full operation to the second half of 2009. Data taking with muons is foreseen to resume in September 2009.

The $5.5 \mathrm{~m}$ long cooling section consists of three absorbers and eight RF cavities encircled by lattice solenoids. The absorber modules are made of a liquid hydrogen 201 container (with relevant safey controls) and a set of two SC focus coils, to provide the required small $\beta$ function inside the absorber. The large $201 \mathrm{MHz}$ RF cavities [10], to handle the extended muon beam, are surrounded by a large SC coil allowing for magnetic coupling with the nearby SC focus coil. The design of the $201 \mathrm{MHz}$ conducting cavities is based on the experience of the US MuCool program.

The cooling process will be studied by varying the relevant parameters, to allow the extrapolation to different cooling channel designs.

\section{The MICE detector system}

The driving design criteria are robustness, in particular of the tracking detectors, to sustain the severe background conditions in the vicinity of RF cavities and redundancy in PID in order to keep beam contaminations $(e, \pi)$ well below $1 \%$ and reduce systematics on the emittance measurements.

\subsection{Spectrometers}

The two identical spectrometers are made of a cylindrical tracker surrounded by a $4 \mathrm{~T}$ solenoid. Each device has five separate coils to provide a very uniform field over the region of interest and ensure a proper optical matching into and out of the cooling cell. The design of the trackers has been optimized to reduce the amount of material in the beam. Each tracker consists of five stations of three planes of $350 \mu \mathrm{m}$ scintillating fibers, rotated by $60^{\circ}$ to resolve spatial ambiguities, in case of multiple hits. The devices are read out by VLPC photodetectors developed for the D0 experiment at the Tevatron [11]. From the hits in the 5 stations, the full trajectory can be reconstructed 
giving the track's $p_{L}, p_{T}$ measurement. Testbeam data have shown a spatial resolution $\sim 440 \mu \mathrm{m}$, that translates to $1.5 \mathrm{MeV} / \mathrm{c}(3.0 \mathrm{MeV} / \mathrm{c})$ for the $p_{T}\left(p_{L}\right)$ resolution of a $200 \mathrm{MeV} / \mathrm{c}$ muon. The spectrometers are expected to be installed in MICE beamline in the first half of 2010, due to some delays in the construction of the tracking solenoids.

\subsection{The PID detector system}

Precision timing measurements ( $\sim 70$ ps resolution) are required to relate the time of a muon to the phase of the RF and simultaneously for particle identification (PID) by time-of-flight (TOF). PID is obtained upstream of the first solenoid by two TOF stations (TOF0/TOF1) and two threshold Cerenkov counters (CKOVa/CKOVb), that will provide $\pi / \mu$ separation up to $300 \mathrm{MeV} / \mathrm{c}$. Downstream the PID is obtained via a further TOF station (TOF2) and calorimeters (EMCAL), to separate muons from decay electrons.

It is not possible to select a single Cerenkov radiator that is sensitive to muons and blind to pions over the entire momentum range. The chosen solution is two different aerogel counters with refractive indices 1.07 and 1.12, each equipped respectively with four 8 " low background EMI9356KA PMTs from the earlier Chooz experiment. Purities better than $99.7 \%$ are thus obtained in the momentum range 210 to $365 \mathrm{MeV} / \mathrm{c}$. At lower momenta, the $\pi / \mu$ separation is obtained by a TOF measurement. All the TOF stations share a common design based on fast 1" scintillator counters along the $\mathrm{X} / \mathrm{Y}$ directions (to increase measurement redundancy) read at both edges by conventional R4998 Hamamatsu photomultipliers ${ }^{1}$. All downstream detectors and the TOF1 station must be shielded against stray magnetic fields (up to 1000-1500 Gauss with a $\leq 400$ Gauss longitudinal component). Two options for the local TOF1/TOF2 shielding are foreseen: in one (for TOF1) a double-sided shielding cage will fully contain the detector, aside a hole for the beam, while in the other (for TOF2) massive soft iron boxes for shielding the PMTs will be used. While the first solution is more elegant and reduces the detector weight, it gives complications for detector access and maintenance.

The TOF stations must sustain a high instantaneous incoming particle rate (up to $1.5 \mathrm{MHz}$ for TOF0). R4998 PMT rate capabilities were tested in the laboratory with a dedicated setup [12] based on a fast laser. The rate capability was increased by the use of an active base.

The downstream calorimeters (EMCAL) consist of a $\mathrm{Pb}$-scintillating fiber calorimeter (KL), of the KLOE type [13], with 1-mm diameter blue scintillating fibers glued between $0.3 \mathrm{~mm}$ thick grooved lead plates ${ }^{2}$ followed by an electron-muon ranger (EMR), made of a $1 \mathrm{~m}^{3}$ fully sensitive segmented scintillator block. This "spaghetti" design for KL offers the possibility of fine sampling and optimal lateral uniformity. The expected resolution $\sigma_{E} \simeq 5 \% / E$ is fully dominated by sampling fluctuations and is linear for electrons or photons in the range 70-300 MeV. EMR will be made with extruded scintillator bars with WLS fibers readout. In the EMCAL while KL will measure electrons, the EMR will measure precisely the muon range.

The TOF0/TOF1 and KL detectors have been installed at RAL in 2008. They have performances compatible with requirements. As an example, figure 3 shows the time difference between

\footnotetext{
${ }^{1} 1$ " linear focussed PMTs, typical gain $G \sim 5.7 \times 10^{6}$ at $\mathrm{B}=0$ Gauss, risetime $0.7 \mathrm{~ns}, \mathrm{TTS} \sim 160 \mathrm{ps}$

${ }^{2}$ since the particle energy is lower than in KLOE, the ratio of fiber to lead has been adjusted by making the lead foils thinner, hence the name Kloe Light or KL in shorter
} 

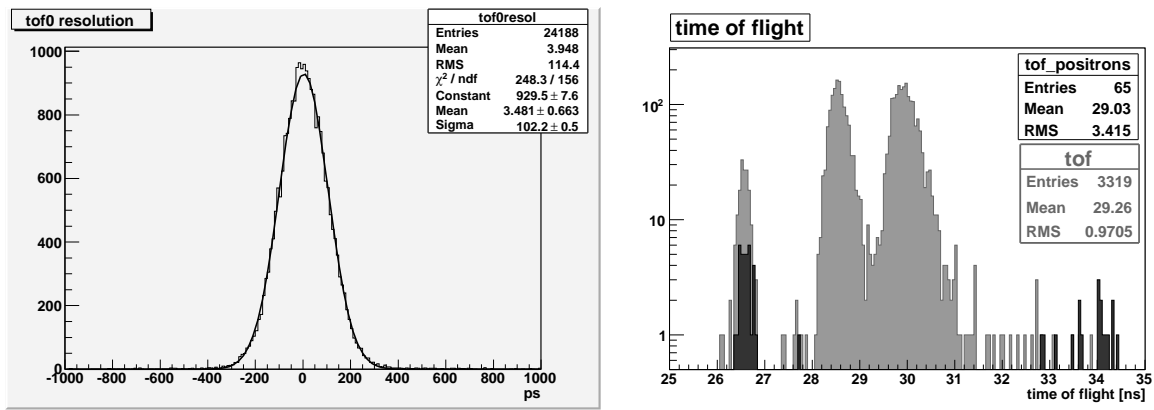

Figure 3: Left: time difference between horizontal/vertical slabs in TOF0. Right: time-of-flight between TOF0 and TOF1 for the so-called positron (black) and pion (grey) beams in logarithmic scale.

the vertical and horizontal slabs in TOF0, that gives a 50/60 ps resolution for a full X-Y TOF station, and the time-of-flight between TOF0 and TOF1 for the so-called positrons or pion beam ${ }^{3}$

\section{Conclusions}

MICE will be accomplished in steps, corresponding mainly to first characterizing the incoming beam and demonstrating the capability to do a high precision measurement of emittance and then to measure the cooling for a variety of experimental situations. The first phase is under way: the beamline has been completed and the upstream PID detectors and KL calorimeter installed. In the near future the following steps are foreseen, aiming at the completion of the experiment in 2012.

\section{References}

[1] D.G. Kosharev, CERN/ISR-DI/74-62 (174).

[2] M.M. Alsharo'a et al., Phys. ReV. ST. Accel. Beams 6,081001 (2003).

[3] A. Blondel et al., CERN-2004-002.

[4] S. Ozaki et al., BNL-52623, June 2001

[5] M. Bonesini, A.Guglielmi Phys. Rep. 433 (2006) 65.

[6] A.N. Skrinsky, V.V. Parkhomchuk Sov. Jour. Nucl. Phys. 12 (1981) 3.

[7] A. Blondel et al., MICE proposal, RAL, 2004;

G. Gregoire et al., MICE Technical Report, RAL, 2005.

[8] C. Booth et al., proceedings EPAC08, EPAC08-WEPP110.

[9] K. Tilley et al., proceedings EPAC08, EPAC08-WEPP122.

[10] D. Li et al., proceedings EPAC08, EPAC08-MOPP098.

[11] A. Bross et al., Nucl. Instr. Meth. A477(2002) 172.

[12] M. Bonesini et al., Nucl. Instr. and Meth. A 572 (2007) 465,

M. Bonesini et al., Nucl. Instr. and Meth. a 567 (2006) 200.

[13] A. Aloisio et al., KLOE coll., Nucl. Inst. and Meth. A 494 (2002), 326.

\footnotetext{
${ }^{3}$ starting from a $300 \mathrm{MeV} / \mathrm{c}$ pion beam, all upstream magnet currents are reduced down to a nominal $100 \mathrm{MeV} / \mathrm{c}$ momentum. At this momentum value only positrons survive.
} 